\title{
Sovrapposizioni. Testo, immagine e fotografia ne Il Giuoco dell'Oca di Edoardo Sanguineti
}

\author{
Henning HufNagel \\ Universität Zürich \\ ORCID: 0000-0002-4850-2523
}

\begin{abstract}
Il saggio legge il romanzo di Sanguineti $\mathrm{Il}$ Giuoco dell'Oca attraverso un nuovo focus: la medialità. Vi si dimostra come, anche senza includere alcuna immagine materiale, esso funzioni come fototesto, anzi costituisca un fototesto di livello potenziato: articolandosi in una serie ecfrastica, mettendo in scena tensioni riflessive tra lingua e immagine, simulando persino il modo di percezione dell'obiettivo fotografico. Attraverso le immagini, Sanguineti contesta non solo i modi narrativi convenzionali, ma spinge la sua sperimentazione anche oltre le avanguardie francesi con le quali dialoga, da Breton ai contemporanei Robbe-Grillet e Sollers.
\end{abstract}

Keywords: narratività, intermedialità, fotografia, Nouveau roman, Tel Quel.

Nel testo del secondo romanzo di Edoardo Sanguineti, Il Giuoco dell'Oca (1967), non è inserita alcuna immagine - fotografica o di altro tipo. Tuttavia non pare troppo audace sostenere che questo testo meriti, come pochi altri, di essere definito come iconotesto e più particolarmente come fototesto. Infatti, Il Giuoco si presenta non come sviluppo narrativo di una trama, bensì come giustapposizione di descrizioni, generalmente ecfrastiche. Evocando così una serie di immagini, perlopiù senza legame, eccetto, al massimo, una similarità di motivi, il testo mostra una struttura non dissimile della mappa del gioco di società eponimo. In III "caselle", Sanguineti descrive figure, oggetti, opere d'arte, ma soprattutto immagini caratterizzate dalla loro (ri)producibilità tecnica. Fra queste figurano, in maniera prominente, fotografie.

L'importanza della fotografia per il romanzo si estende su più livelli: anche quando Sanguineti descrive degli spazi, questi sono a volte strutturati da fotografie, come si legge già nel secondo capitolo, dove una "parete» non fa che da supporto a «otto o nove» fotografie (II). E quando descrive delle persone, spesso queste si rivelano come immagini fotografiche di persone, inserite in contesti enigmatici dove i corpi si frammentano mentre lo spazio si appiattisce nella bidimensionalità di un'immagine; oppure, data l'eterogeneità degli elementi evocati insieme, di un collage, come nel capitolo CV: «Poi c'è la donna, di sole gambe, con le calze bianche. Le hanno fotografate in piedi, quelle gambe, con le calze» (224). Ci sono persino fotografie all'interno di altre immagini, come nei silkscreen paintings di Robert Rauschenberg descrit- 
te nelle caselle LXII e LXXIII che integrano riproduzioni fotografiche in un contesto pittorico.

Poiché le fotografie descritte sono usualmente impregnate di segni linguistici ${ }^{I}$, quasi come comic strips (secondo tipo di immagine riproducibile che Sanguineti non si stanca di evocare ${ }^{2}$ ), presentandosi spesso come provenienti da fogli di giornale, esse mostrano esplicitamente un'interazione tra parola e immagine. Nel capitolo LXXIV queste interazioni sono persino potenziate: il testo verbalizza una fotografia di una donna che mostra delle fotografie applicate a un cartellone, accompagnate da parole.

Il mio contributo analizza come, in questa maniera, il romanzo di Sanguineti funzioni come un fototesto di secondo o persino terzo livello, producendo continuamente riflessioni sulle dinamiche tra lingua e immagine, visione e narrazione, riproduzione meccanica ed esperienza organica. Così, accanto alle giustapposizioni delle descrizioni, si constatano multiple sovrapposizioni - mediali e riflessive - al loro interno: le «superpositions / d'images de catalogue» (7) che Sanguineti annuncia nell'epigrafe del suo romanzo.

In ciò che segue vengono rivisitate le nozioni teoriche dell'icono- e fototesto per precisare lo statuto intermediale del Giuoco. Si chiarisce in seguito come la "svolta ecfrastica" del romanzo stia alla base della sua contestazione neoavanguardistica dei modi convenzionali del narrare, tenendo conto del suo contesto francese, da Breton a Robbe-Grillet e Sollers. Spicca qui il dialogo che si instaura con il fototesto Nadja. Viene quindi mappata la presenza della fotografia nel Giuoco e se ne studia la relazione con altre forme artistiche, soprattutto il cinema, arte narrativa che appunto si basa sulla sequenza di immagini fotografiche. Infine, si sostiene che il romanzo di Sanguineti è un fototesto in senso fondamentale, perché non solo evoca delle immagini fotografiche, ma simula nelle sue descrizioni verbali il modo di percezione dell'obiettivo fotografico.

A prima vista, la definizione del concetto di iconotesto, e sulla sua scia, di fototesto, non sembra presentare ambiguità. Un iconotesto è un testo a doppio codice, nella cui pagina sono presenti sia parole che immagini, un com-

\footnotetext{
I Per es. nei capitoli II, XXXII, XXXIV, LXXIV. Anche nei quadri di Rauschenberg descritti, alcune delle foto inserite comportano parole, ugualmente riportate da Sanguineti.

2 Per es. nel capitolo III è descritto come dalla bocca del protagonista esce una nuvola bianca che contiene parole in lettere maiuscole. Infatti, indicando una prima dimensione di interazione tra parola e immagine nel Giuoco, c'è da sottolineare che sono generalmente i segni linguistici a identificare una scena descritta come immagine fumettistica, attraverso le maiuscole citate o le onomatopee, per es. «VROAAWIIII!» (II9) o «KA-FOOM» (I85). Sanguineti evoca anche personaggi dei fumetti nominandoli, tra gli altri, Wonder Woman (23) e Supergirl (236).
} 
positum mixtum dotato di una dimensione verbale e una dimensione visuale. Il fototesto, di conseguenza, non è altro che un caso specifico dell'iconotesto in cui la dimensione visuale è costituita da fotografie, combinate, in varie forme di layout, con un testo verbale ${ }^{3}$. Come esempi si possono citare i libri di W.G. Sebald, la Lettura di un’mmagine (1975) di Lalla Romano o ancora i "ciné-romans», che combinano testi da copione e film stills, di Alain Robbe-Grillet; Bruges-la-morte (I892) di Georges Rodenbach è l'opera-fondatrice del genere. La questione del predominio di una delle dimensioni è secondaria. A partire da un'osservazione di Andrea Cortelessa, Maria Rizzarelli ha sottolineato che «non è la quantità di immagini a definire lo statuto fototestuale, quanto la stretta relazione che si stabilisce fra parole e immagini» (2019: 50). A questa riflessione è comunque sotteso il presupposto che ce ne sia almeno una.

Tuttavia Peter Wagner, teorico dell'iconotesto che nella critica passa per uno dei più influenti e la cui definizione è stata considerata la "più sintetica e valida» (Cometa 20I6: 70), è meno perentorio. Questo aspetto viene in genere tralasciato, visto che si tende a citare la sua definizione senza contesto. Wagner scrive: «[...] iconotext refers to an artifact in which the verbal and the visual signs mingle to produce rhetoric that depends on the co-presence of words and images» (I996: I6). In un primo momento, questa definizione sembra concordare perfettamente con quella sopra formulata, ma guardando più da vicino si scopre che «co-presence», per Wagner, significa non compresenza materiale, sulla pagina, bensì nella mente dei lettori. Egli applica il termine 'iconotesto' "not only to works which really show the interpenetration of words and images in a concrete sense [...] but also to such art works in which one medium is only implied (e.g., the reference to a painting in a fictional text)» (I6).

Seguendo dunque Wagner tel quel, parlare del romanzo di Sanguineti come fototesto perderebbe quasi ogni intento provocatorio (se non verso quella critica che legge Wagner in modo riduttivo), questo tuttavia significherebbe sottovalutare il romanzo: le modalità con cui continuamente mette in scena e simula immagini verbalmente senza mai mostrarne alcuna costituiscono infatti una sfida a concezioni consuete di genus e media. Wagner concepisce il concetto in un senso così ampio, vedendo già nella designazione di un'immagine o nell'allusione a essa una condizione sufficiente di iconotestualità, che rischia di far perdere al concetto il suo profilo analitico

3 A tal proposito si rimanda al contributo teorico recentemente offerto da Michele Cometa (20I6); una concezione analoga sta alla base anche della «mappa del fototesto italiano contemporaneo» di Maria Rizzarelli (2019). La copresenza materiale di immagine e testo era già il principio-base di Michael Nerlich (1990) e di Alain Montandon (1990) quando forgiarono il termine di iconotesto, da Nerlich applicato proprio a una combinazione di testo verbale e fotografie. 
nonché di ampliare il suo corpus quasi illimitatamente. Invece è opportuno, in considerazione dei loro diversi meccanismi di significazione, differenziare tra opere che combinano materialmente dei media differenti (come Bruges-la-morte); che tematizzano prodotti di altri media citandoli, cioè nominandoli o facendovi allusione (per esempio, secondo Wagner, Moby Dick che si riferisce implicitamente a Le serment des Horaces di Jacques-Louis David [I5]); e infine quelle opere che simulano in un medium le modalità di un altro ${ }^{4}$.

Si può constatare così una gradazione nel carattere "iconico" di un testo: la seconda categoria presenta il grado più debole, poiché opera solo con il codice linguistico, e la prima quello più forte, in quanto attiva direttamente nei lettori i modi di ricezione di immagini accanto a quelli di un testo verbale. La terza categoria, di grado intermedio, però, sembra quella più raffinata perché i lettori, recependo il testo dapprima come testo verbale, vengono spinti ad adoperare simultaneamente un secondo set di modi di ricezione, quello iconico, all'interno del testo verbale stesso.

La nostra ipotesi è che nel Giuoco occorrono fenomeni della seconda e della terza categoria. Sono così diffusi e strutturano il testo in un modo così fondamentale che ne fanno quello che chiamo un fototesto di livello potenziato. Infatti, la simulazione "altermediale" - la simulazione, in un medium, delle modalità di un altro - sta alla base della sfida estetica del testo di Sanguineti. Con il suo ostentato sottotitolo di «romanzo», l'opera crea aspettative di narratività che il testo non fa che frustrare, presentando spunti di trama sempre nuovi a cui in genere non segue nulla, sviluppandosi invece in una successione di descrizioni e di immagini verbalizzate - una successione in più aleatoria, instabile, se prendiamo sul serio le indicazioni sulla quarta di copertina, che invita a «giocare» al romanzo, stabilendo l'ordine di lettura tramite due dadi.

In più, il fatto che l'autoproclamato romanzo si rifiuti di raccontare corrisponde all'evocazione ubiqua delle immagini che costantemente si sottraggono, rimanendo sempre materialmente assenti, creando una sensazione di disorientamento e di mancanza: Il Giuoco dell'Oca è sia un romanzo che un fototesto in uno strano stato di entre-deux, in un limbo al quale corrisponde infine la figura dell'io narrante, introdotto fin dalla prima riga, giacente nella bara, morto, eppure sveglio e attento, eloquente nonché attivo osservatore. La questione della fototestualità del Giuoco è dunque intimamente legata alla contestazione neoavanguardistica dei modi convenzionali del narrare.

4 Riprendo la distinzione introdotta da Irina Rajewsky tra «Medienkombination» e «intermediale Bezüge» (2002: I5-I8), modificando e articolando a mia volta questa seconda categoria. 
Tra le doléances che la neoavanguardia italiana formula contro lo stato della letteratura italiana degli anni ' 60 (per alludere a un noto saggio di Renato Barilli dagli accenti prerivoluzionari), ne spiccano due, come si sa: il provincialismo e, connessa ad esso, l'arretratezza dei moduli narrativi dominanti, (neo)realistici, eredità, in sostanza, ancora dell'8oo. Nel Giuoco, Sanguineti affronta queste insufficienze fin dalla dedica:

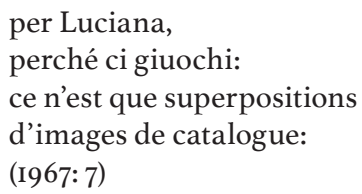

Invece di invitare sua moglie alla lettura, guidata magari da un narratore con funzione ordinatrice, Sanguineti la invita al gioco, implicando dunque un ruolo attivo della ricevente, muovendosi sulla scia dell'«opera in movimento» celebrata da Umberto Eco fin dal 1959 (2013: 749).

Le due righe che seguono in francese specificano l'attacco avanguardistico contro i modi realistici. Da un lato, naturalmente, Sanguineti annuncia il ruolo fondamentale della dimensione visiva e iconica per il testo. D'altro lato, si rifà alle prime avanguardie internazionali. Infatti, l'autore cita dal Manifeste du surréalisme (1924), e proprio dalla sezione in cui Breton fa il «procès de l'attitude réaliste», denunciando l'«abondance des romans» che si perderebbe in notazioni contingenti, di «caractère circonstanciel, inutilement particulier» (1972: I7-I8). In breve, Breton se la prende con quello che Roland Barthes, nel 1968, chiamerà l'«effet de réel». E se, nella sua analisi, Barthes focalizza i passaggi descrittivi del romanzo realista, Breton, nella sua polemica, ha la stessa mira:

Et les descriptions! Rien n'est comparable au néant de celles-ci; ce n'est que superpositions d'images de catalogue, l'auteur en prend de plus en plus à son aise, il saisit l'occasion de me glisser ses cartes postales, il cherche à me faire tomber d'accord avec lui sur des lieux communs (I8).

Breton critica il carattere di cliché, di immagine stereotipica, prefabbricata delle descrizioni realiste, che, secondo lui, mancano di originalità e di immaginazione. Si può aggiungere che, in questa maniera, esse falliscono anche in quello che si propongono di fare, ossia di cogliere direttamente un aspetto del reale. Sanguineti riprende la formula dispregiativa delle «images de catalogue», ma affermativamente. Non rigetta dunque la posizione di 
Breton, ma la supera ironicamente, tenendo conto delle aporie delle avanguardie storiche. Di queste ci si rende conto analizzando Nadja (I928).

Questo celebre fototesto è concepito come l'esecuzione fedele delle prescrizioni del manifesto, come Breton sottolinea nella sua prefazione tardiva del I962, spiegando che «l'abondante illustration photographique a pour objet d'éliminer toute description - celle-ci frappée d'inanité dans le $\mathrm{Ma}$ nifeste du surréalisme» (I964: 6). Leggendo Nadja si deve però constatare che questo non è affatto il caso. Se Breton suggerisce di eliminare tutte le descrizioni perché tendono al cliché, non significa che il testo possa abbandonare ogni carattere descrittivo. Le foto inserite - dei disegni fatti da Nadja, ma anche di località e oggetti - sono infatti accompagnate da un commento verbale, in didascalia e nel testo, che ne accentua i dettagli. Le descrizioni realiste non sono dunque semplicemente cancellate, bensì sostituite da descrizioni, per giustapposizione, ecfrastiche. Non si descrive più un presupposto reale, ma la sua immagine. Sanguineti procede esplicitamente in questa maniera.

Comparate alle descrizioni realiste, le fotografie promettono di stabilire una referenza più diretta (e verace) al reale a causa del loro carattere indessicale, nel senso di C.S. Peirce di una contiguità attuale, causale tra segno e mondo (Metz I990: I56), essendo l'immagine fotografica (almeno prima dell'epoca digitale) la traccia chimica della luce riflessa dall'oggetto davanti alla fotocamera (Horstkotte-Pedri 2008: I3). Ciononostante, Breton stesso cede, forse involontariamente, all'inevitabilità della descrizione quando presenta un disegno particolarmente denso in cui si sovrappongono facce, figure e oggetti:

Dans celui-ci [questo disegno] on distingue [segue una lunga lista di forme, che si conclude su] le visage d'une autre femme, un serpent, plusieurs autres serpents, un cœur, une sorte de tête de bœuf ou de buffle, les branches de l'arbre du bien et du mal et une vingtaine d'autres éléments que la reproduction laisse un peu de côté mais qui en font un vrai bouclier d'Achille (Breton I964: I43-I46).

Breton constata dapprima i limiti della fotografia che non coglie tutti gli elementi che l'interpretazione verbale può rilevare, per poi finire la sua descrizione riferendosi a questo disegno proprio con il nome dell'oggetto arci-paradigmatico dell'ecfrasi, conosciuto da tutti i manuali di retorica, tratto dal canto XVIII dell'Iliade.

Sanguineti trae da questo le opportune conseguenze. Se ogni descrizione tende al cliché, ma è inevitabile, meglio partire consciamente dai clichés, dalle immagini prefabbricate - figuralmente e letteralmente -, abbandonando ogni miraggio di referenza diretta al reale come lo nutriva il realismo, ma scartando anche ogni tentativo, aiutato, magari, dalla referenzialità fo- 
tografica, di accedere alla «vie réelle» interiore, "autentica", che il Manifeste du surréalisme invocava enfaticamente (Breton 1972: 15).

Il capitolo LXXIX è una vera e propria mise en abyme di questo procedimento, presentando un readymade testuale, completamente enigmatico alla prima lettura. È tuttavia possibile identificarne gli elementi, cosa che permette di evidenziare il modus operandi di Sanguineti, svelando il loro significato poetologico. In un primo momento Sanguineti descrive quello che si rivela come la confezione di un giocattolo, una trottola musicale. Vi si vede una ragazza in abito rosso accanto al «titolo» perforato «VOICEDBOPPERTOP (V rosso, O giallo, I rosa, C rosso, E verde, D viola [...])» (I967: I68).

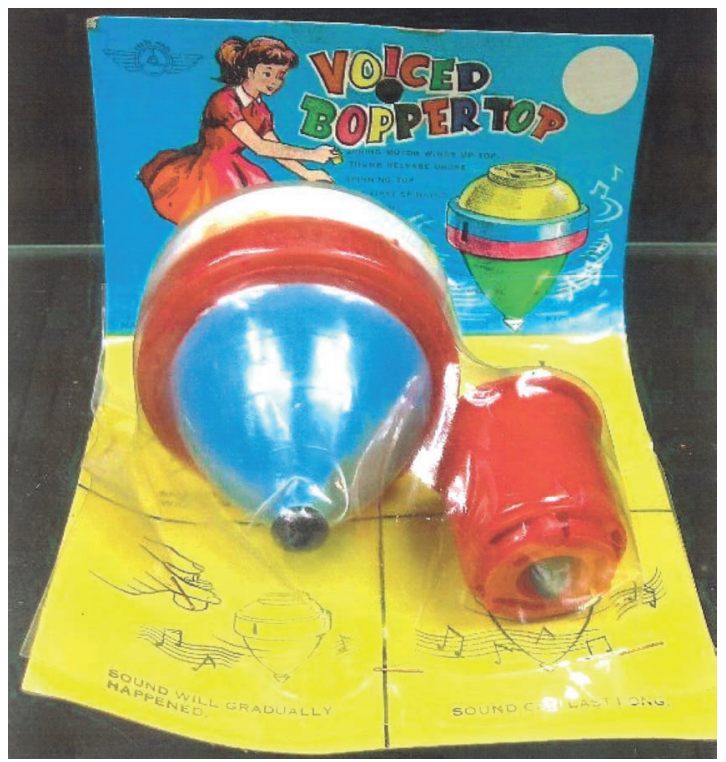

Fig. I: Voiced Bopper Top, foto: dug4you, da Ebay.com.

Per le sue lettere colorate, questo objet trouvé appare, nella verbalizzazione di Sanguineti, come un'attualizzazione del sonetto metapoetico Voyelles di Arthur Rimbaud - un'attualizzazione giustamente sotto le condizioni banalizzanti della riproducibilità tecnica industriale. In seguito, vi si applica un secondo pezzo di carta, un «tagliandino», creando - si noti la parola-chiave nel passo citato - una sovrapposizione:

Nel buco della scena della bambina rossa ci metto il tagliandino con il buco. Faccio coincidere i due buchi, li sovrappongo. Il tagliandino dice di guardarci il mondo. Dice look through this hole at the world. Il tagliandino arriva da Praga, via Milano. Ci guardo il mondo (169). 
Questo «tagliandino» non è altro che una delle carte che formano le Fifty-eight propositions for One Page (1965) dell'artista Fluxus Ben Vautier, ciascuna stampata con una breve istruzione più o meno conturbante (fino $\mathrm{a}$ «swallow this page») .

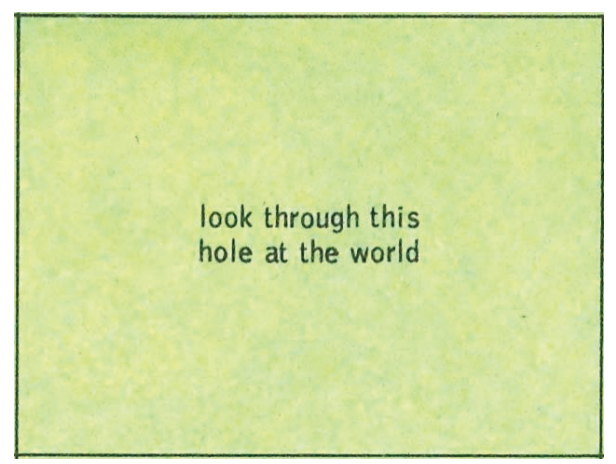

Fig. 2: Ben Vautier, Fifty-eight propositions for One Page (particolare). (C) ProLitteris, foto: Daniel Thierstein Antiquariat Bern, catalogo Archiv «edition et».

Quello che importa fondamentalmente nel nostro contesto è che, con questa sovrapposizione, Sanguineti crea un dispositivo visivo che è una mise en abyme dell'intero Giuoco. Smentendo ogni interiorità, l'io narrante percepisce il mondo attraverso strati multipli di artefatti, di oggetti, industriali e artistici, entrambi tecnicamente prodotti. Questo dispositivo è a sua volta tecnico, fotografico anzi. Il buco nella carta evoca infatti la fotocamera primitiva della stenoscopia: mediando la percezione, l'oggetto si trasforma in obiettivo.

Va tuttavia osservato che la carta di Vautier non è affatto perforata; la sua istruzione è paradossale (come altre, per esempio, «I don't propose you do anything with this page»), il buco è immaginario, e lo sguardo non arriva mai fino al «mondo», ma resta attaccato all'artefatto.

Conforme a questo dispositivo, Sanguineti segue una strategia doppia. Primo, si serve di immagini prefabbricate nei due sensi: accanto al patrimonio highbrow (come la Nave dei folli di Bosch, alla casella LXVIII), in maniera dominante, di immagini della pop culture, di fumetti, pubblicità (1967: I28), di sue figure "iconiche" come Marilyn Monroe e appunto di fotografie, arte middlebrow, secondo la formula di Bourdieu (1965) - fotografie non ar-

5 Furono pubblicate in Fluxus newspaper $\mathrm{n}^{\circ} 7$ ( $\mathrm{I}^{\circ}$ febbraio I966) su una stessa pagina non numerata, in modo da essere tagliate dai lettori. Nelle collezioni del MoMA di New York si trova sia la pagina intera che un esemplare di carte separate (https://www.moma.org/collection/ works/I379I7 e I3I856, consultati il I8.06.202I). 
tistiche, ma giornalistiche (Sanguineti I967: II, I57, I76), da cartolina (I99) o private (96).

Se Breton opta per le fotografie, preferendo le immagini alle parole, proprio per il loro carattere indessicale, Sanguineti invece riflette sull'artificialità della medializzazione iconica. Problematizzando la promessa fotografica di referenza, le foto di Sanguineti sono spesso caratterizzate da una forte dose di messa in scena, provenendo, per esempio, da un set cinematografico (75) oppure persino estratte dal rullino di un film e mostrate come «diapositiv[e]» in un teatro (85).

Breton voleva superare il realismo eliminando la descrizione, perché inautentica, e sostituendola con le immagini fotografiche. Sanguineti supera tanto il realismo quanto Breton eliminando la narrazione e sostituendola con la descrizione di immagini, smascherando le loro promesse di contatto col reale: non si scappa dai media.

Tale "finzionalizzazione" della fotografia conduce alla seconda strategia annunciata sopra: il Giuoco si presenta continuamente e a tutti i suoi livelli come finzione, espone costantemente la sua artificialità. Ricorre lungo tutto il testo un'isotopia, a volte metaforica, a volte no, del teatro, del palco e della scena (per es. I3, I7, 27-28, 69, 80, I06, I36, I40, I72, I86). Ci sono personaggi che invece di parlare, cantano, presentando una forma esteticamente sovradeterminata, artificiale del discorso (IO, I45, I86). E quando parlano, il loro discorso è regolarmente "medializzato", cioè identificato come testo scritto, indicato dalle lettere maiuscole o corsive in cui appare in pagina (per es. II, I3, I85, 2I3). A volte questa scrittura è esplicitamente messa sullo stesso piano dei personaggi che l'enunciano. Così, in un primo momento, seguendo aspettative consuete di lettura, questi personaggi appaiono come una rappresentazione verbale di esseri umani - esseri umani che parlano tra di loro. In seguito, però, diventando manifesto il carattere scritturale del loro discorso, si rivelano anch'essi medializzati: quasi personaggi fumettistici con le loro nuvolette, sono una rappresentazione verbale di una rappresentazione figurata.

\section{3}

Sanguineti usa modi persino più vistosi per segnalare l'artificialità del suo romanzo e dunque distruggere quell'illusione di un mondo plausibile alla quale mira la narrazione realista. Lautore semina incoerenze fin dal primo capitolo, in cui i personaggi sono introdotti prima come «fatti di legno, un po' come nei tiri al bersaglio» (9), poi si animano per infine ridiventare di legno qualche riga più sotto, - questo fin dall'io narrante, presente in quasi ogni casella, ma completamente insostanziale, in continua metamorfosi, per lo più anonimo o chiamato a turno «Bruce», «Greg», «Pierre» 
(I27, I6o, 2I4), perfino triplicandosi, cambiando sesso ed età, in uno stesso capitolo $(225,233)$.

Queste tecniche si riferiscono a un secondo contesto avanguardistico e francese, più vicino nel tempo, e direttamente legato alla contestazione del narrare tradizionale, convenzionalmente realista. Ci si riferisce al nouveau roman di Robbe-Grillet, proposto come modello da Barilli ${ }^{6}$. Più specificamente, Sanguineti si rifà al romanzo Dans le labyrinthe (1959) dove i personaggi si trasformano ripetutamente in immagini e viceversa, dando alla formula «école du regard» una dimensione mediale. Il suo incipit apre persino, trasposto in modo assai fedele, Il Giuoco dell'Oca: «Je suis seul ici, maintenant, bien à l'abri» (Robbe-Grillet 1959: 9) - «Ci sono io, per intanto. Sto dentro la mia grande bara» (Sanguineti 1967: 9)7

Sanguineti radicalizza tuttavia l'approccio di Robbe-Grillet, in primo luogo attraverso la moltiplicazione delle immagini, analoga alla sua scelta di immagini tecnicamente (ri)prodotte. Alla base della rivoluzione del narrare operata dal nouveau roman sta, nelle parole di Rainer Warning, la trasformazione della narrazione in una sequenza di descrizioni (200I: I79; I999: 83), sovrapponendo alla sequenzialità, sintagmatica, la serialità, paradigmatica; cioè spostando la dominanza dalla contiguità alla similarità. Sanguineti potenzia la dimensione paradigmatica riducendo le similarità e indebolendone la coerenza: se in Robbe-Grillet tornano ossessivamente sempre gli stessi elementi, in Sanguineti ne appaiono sempre di nuovi, con ogni nuova immagine. L'io insostanziale ne è l'emblema ${ }^{8}$.

\section{4}

Ciò conduce alla radicalizzazione di un secondo modo contemporaneo di contestazione del narrare tradizionale: quello che viene dal gruppo Tel Quel e si manifesta nel romanzo intitolato (paradossalmente) Drame (I965) di Philippe Sollers (e questo "dramma" trova una sua eco già nell'isotopia del teatro sopra accennata). Drame racconta come un protagonista - a volte «il», a volte «je», semplice pronome come nel Giuoco, benché coerente - ricomincia continuamente a scrivere, nel tentativo di raccontare una «véritable

6 Cfr. Ó Ceallacháin (2013). Il Giuoco gli pare però distante da Robbe-Grillet.

7 Per una comparazione estesa dei due testi cfr. Bongiorno 20II, che sottolinea anche il parallelismo degli incipit. Anche Hempfer (1999: I72) connette i due testi.

8 Anche indebolendo coerenza e similarità, Sanguineti non le abbandona al punto di dissolvere il susseguirsi di elementi in un caos. Tornano motivi, come accennato, quale la scena, la bara o ancora il tuffo nell'acqua, gli occhiali e la mummia, e particolarmente nella seconda parte del romanzo alcune caselle formano micro-sequenze, soprattutto trilogie, in analogia alla triplicazione dell'io (per es. LXXXIX, XCII, XCVI; C, CIV, CVIII; CII, CV, CIX). Ma sono legami molto più sottili. 
histoire», opposta a una «fable commode» (II, 6I). Diventa ben presto chiaro che questa «fable» non è altro che la storia "falsa", "illusionistica", da romanzo tradizionale, mentre la «véritable histoire» consiste nella notazione dell'atto di scrittura stesso: «je vais raconter exactement le trajet des mots sur la page - exactement, rien d'autre, rien de plus» (I47). Drame si presenta dunque come fondamentalmente autoriflessivo, cioè, in termini narratologici, come un discours senza histoire ${ }^{9}$.

Anche Il Giuoco conosce l'autoreferenzialità, benché non in modo così esclusivo. Tematizza regolarmente la produzione dell'artefatto, ed è significativo che, a differenza di Drame, evoca processi sia linguistici che iconici: l'io narrante è presentato nell'atto di scrittura, come un San Geronimo tra candela e teschio, e scrive persino l'ultima parola del romanzo che, autoriflessivamente, è «FINE» (Sanguineti I967: 48, 238). Ci si vede «disegna[re]» (69) personaggi e persino se stesso - «I due io [...] li ho fatti senza staccare la penna dal foglio» - trovandosi metaletticamente sullo stesso piano di questo suo disegno come in un'opera di M.C. Escher (234). È inclusa in questo processo anche la fotografia: l'io è fotografato (25-26) e scatta lui stesso fotografie in un «teatro di posa» (I99), e tutto il capitolo LVIII consiste in istruzioni per fare un collage, oscillando tra l'organico e il meccanico, tra il tableau vivant e i pezzi di carta, collage che alla fine viene fotografato. Il capitolo è un buon esempio per il genere di sovrapposizioni mediali operate nel Giuoco:

Si prende una ragazza, una qualunque, lí a caso. Si mette la ragazza, lí di fronte a noi, nuda, seduta sopra uno sgabello di legno, con la faccia tutta in ombra, le ginocchia in piena luce, le mani sui fianchi. Si tagliano i piedi. Si butta un resto di tempio ionico, lí sopra la ragazza [...]. Si dice alla ragazza che deve girarsi tutta, lei, lí di schiena, in piedi. Si tagliano i piedi. Si taglia tutto quello che c'è, dalle ginocchia in giù [...]. Si buttano due che guardano il tramonto, un uomo e una donna, sulla riva del mare [...], con il vento che soffia dentro i capelli della donna. Poi si fotografa tutto. CAMERA: Pentax $\mathrm{H}_{3}$, with Super-Takumar $f / \mathrm{I} .8$ lens. EXPOSURE: at $f / 2.8$ shutter speed varied. FILM: Kodachrome Type B. LIGHTING: two $3200 \mathrm{~K}$ floods bounced (I23-I25).

Il Giuoco condivide con Drame anche il gesto del ricominciare sempre di nuovo: i capitoli non connessi tra di loro sembrano cominciare sempre una nuova storia. Ma si mostra già lì la differenza tra i due testi - e la radicalizzazione operata da Sanguineti. Mentre lo scrivente di Sollers ricomincia sempre lo stesso progetto - scrivere la «véritable histoire» -, l'istanza enun-

9 Cfr. Hempfer (I999: I65-I67). Hempfer analizza poi le relazioni tra Drame e Il Giuoco sotto il segno dell'autoreferenzialità performativa, vedendo nel Giuoco un'ironizzazione di Drame (I7I-I73). Considerando la dimensione delle immagini, vi si vede piuttosto una radicalizzazione. 
ciativa di Sanguineti comincia sempre qualcosa di diverso, risultando nella messa in scena di potenzialità: storie possibili però mai realizzate. Qui sono centrali le descrizioni di immagini perché bloccano, per così dire, il tempo, impedendo l'avviamento di una storia. Come sottolinea Genette, la narrazione, basata sulla sequenza di azioni ed eventi, è connessa alla dimensione temporale, mentre la descrizione, nella narratologia, è generalmente associata a una sospensione del tempo (1966: 158).

Ora, se la contestazione del narrare tradizionale di Sollers consiste nella produzione di un discours senza histoire, Sanguineti lo sorpassa ludicamente, visto che Il Giuoco dell'oca non solo non dispone più di nessuna histoire fissabile (per mancanza di una sequenza di eventi trasversale, e anche all'interno di molte caselle, bloccata dalla descrizione di immagini). Ma - e per questo mi sembra importante prendere alla lettera il titolo del romanzo -, in quanto gioco, proponendo un ordine di lettura aleatorio, non dispone più neanche di discours fisso. Questa sfida al discours è legata alla descrizione di immagini in quanto base dell'articolazione del romanzo come struttura iconica, spaziale, di caselle da mappa del gioco dell'oca.

\section{5}

La discussione della narratività specifica del Giuoco ha mostrato quanto essa si fondi sulla referenza alle immagini e alla fotografia in particolare, senza mai trascendere i limiti del testo composto di segni linguistici. Per avviare la riflessione dei lettori su questa discrepanza mediale, Sanguineti inserisce questi riferimenti in un campo di tensioni che rispecchiano le dinamiche tra lingua e immagine, narrazione e visione, dal momento che (facendo eco a Lessing) esse appaiono in ultima istanza come tensioni tra spazio e tempo: tensioni fra l'organico e il meccanico, incarnate nell'io-mummia putrescente da un lato e nelle immagini di corpi nettamente tagliate nei collages dall'altro, ma soprattutto tensioni fra movimento e stasi, incarnate nella processualità dell'opera in corso di produzione e la rappresentazione di immagini prefabbricate, preesistenti, nonché nell'apparente sospensione del tempo in molte delle «scene» descritte (per es. Sanguineti I967: 77, I40) e la messa in movimento di immagini descritte in precedenza, come avviene nel capitolo XCI per Tracer (1963) di Rauschenberg, già descritto nel capitolo LXXIII.

Sanguineti introduce tali tensioni all'interno delle immagini stesse, contrapponendo il cinema alle altre arti iconiche. Basato sulla sequenzialità di immagini, il film è l'arte narrativa par excellence fra le arti iconiche. Non a caso le uniche volte che compare il verbo "raccontare" nel Giuoco, questo racconto è subito doppiato nel medium filmico (I05, II2). Come già accennato, Sanguineti sospende il susseguirsi continuo dei fotogrammi isolando singo- 
le «diapositive». Proiettandole sulla bara, crea invece sovrapposizioni, cioè similarità all'interno di un paradigma. Nel caso specifico sono le similarità fra l'io nella sua bara e il personaggio principale del film Vampyr (I930) che guarda fuori dal feretro $(85)^{1 \mathrm{O}}$.

Lo stesso meccanismo di sostituzione della sequenzialità con la similarità paradigmatica si nota nella casella XXXIV, evocando la scena "iconica" da The Seven Year Itch (1955): Marilyn Monroe nell'abito bianco sulla griglia della metropolitana. L'immagine fotografica è presentata come parte di una specie di giocattolo con cui si può ricreare la scena famosa (trasformata così quasi in «opera in movimento»), sempre di nuovo, alzando la gonna sempre di più, e infatti viene ripetuta varie volte nella casella, già subordinando la sequenza al paradigma.

In un rovesciamento caratteristico, Sanguineti parla dell'«aria calda» che salirebbe dalla griglia, mentre nel film la giovane donna si mette lì proprio per sentire il soffio fresco del treno che passa. Sanguineti invece evoca il fiato, ardente di desiderio, dello spettatore/lettore: «tu [che] soffi forte con la bocca, con il fiato caldo» (75). Così, anziché sviluppare la trama di quel film, ne evoca un altro: Der Blaue Engel (1930) dove il professor Rath soffia su una cartolina alzando le piume che vi sono applicate a mo' di gonna, scoprendo le gambe di Marlene Dietrich.

\section{6}

Si è già evidenziata la natura fotografica del dispositivo visivo del capitolo LXXIX. Resta da mostrare come, conformi a tale dispositivo, le descrizioni del Giuoco simulano il modo di percezione fotografica. Infatti, questa simulazione di altermedialità sta alla base del loro carattere straniante. Si può citare la loro stasi atemporale accennata prima. Si può aggiungere il fatto che i rumori sono in genere assenti. Questo colpisce particolarmente nel caso della donna col cartellone: dai frammenti di parole citate («GEORG», «PAU», «RINGO») si capisce che si tratta di una fan dei Beatles «tutta appassionata» (I58). Ma in contrasto con le folle di ragazze strillanti a ogni apparizione del gruppo testimoniate dai documentari, qui non c'è alcun segno della sua voce. Si aggiunge infine l'uso della parola «poi»: Sanguineti fa oscillare il suo senso tra il temporale e lo spaziale, come sinonimo di "accanto" $(60,69)$, ribaltando apparenti sequenze di azioni nella simultaneità di immagini. Ma tutti questi elementi valgono per le immagini in generale.

Ciò che rende fotografiche le sue descrizioni non è neanche un elemento associato comunemente a una scrittura fotografica (e più spesso ancora

IO Allasia (20I7: 54, 63-65) sottolinea il significato del film per Sanguineti, in generale, e per l'incipit del Giuoco, in particolare. 
filmica), cioè un'impressione di neutralità e oggettività (Hertrampf 20II: 87), risultando in notazioni che si presentano come prive di ogni interpretazione umana, come si è detto di molte descrizioni di Robbe-Grillet. In parte le descrizioni del Giuoco presentano questo aspetto (per esempio notando semplicemente «PAU», invece di esplicitare «PAUL, ma la L è nascosta»), in parte esso è subito decostruito attraverso l'implicazione quasi ubiqua dell'io e/o del tu, "organico", con i suoi desideri. Le descrizioni del Giuoco sono testualità fotografiche perché presentano tutti gli elementi evocati sullo stesso piano, bidimensionale, senza prospettiva, indifferente al loro genere, se corpo, oggetto, immagine, scrittura.

Queste descrizioni dunque non corrispondono a uno sguardo umano filtrato da una macchina fotografica, come nelle descrizioni di Robbe-Grillet. Il loro procedere è più radicale: non corrispondono all'apparato percettivo umano (che genera la percezione della profondità nel cervello), ma proprio all'apparato percettivo, meccanico, dell'obiettivo fotografico, per il quale tutti gli elementi sono uguali in quanto tutti indistintamente impressioni di luce ${ }^{\mathrm{II}}$.

Come esempio conclusivo per questa simulazione dell'obiettivo fotografico ho scelto la casella LXXXIII e se ne potrebbero facilmente aggiungere altre. Questa casella verbalizza un'altra fotografia di Marilyn Monroe e vi confluiscono anche altri aspetti della narratività del Giuoco già evocati, come la referenza al cinema senza che ne derivi una trama filmica, il canto come discorso sovradeterminato, la sovrapposizione dei codici nelle immagini impregnate di segni scritturali e ancora la tensione fra l'organico e il meccanico. È stato possibile individuare la fotografia concreta alla sua base: una delle moltissime scattate al promotion party nella villa del musicista Ray Anthony a Los Angeles nel 1952, promotion sia della nuova canzone Marilyn che della fresca vedette del film Niagara di cui indossa l'abito magenta ${ }^{12}$. La si mostra qui perché il confronto diretto con l'immagine rende immediatamente evidente il carattere fotografico della descrizione.

II Elisa Sotgiu ha notato perspicacemente il carattere "piatto" delle descrizioni nel Giuoco, osservando come le evocazioni della pop-art vadano di pari passo con la trasposizione dei «presupposti del loro operare», cioè «il montaggio senza prospettiva dei collages». Come esempio cita la casella LXXXVIII che verbalizza il collage di Richard Hamilton Just what is it that makes today's homes so different, so appealing? (1956), mostrando una coppia nuda in una «sghemba stanza» (20I6: I48). Giustamente questo aggettivo che rinvia all'illusione prospettica mi sembra indicare che l'osservazione è giusta, ma la riflessione non guarda abbastanza lontano. Sanguineti non simula verbalmente il collage in quanto collage, cioè giustapposizione di materiali bidimensionali (che poi creano un'illusione di profondità), ma, introducendo un ulteriore strato mediale, lo mostra come lo vede l'obiettivo fotografico. In più, questo procedere non è limitato alle caselle che evocano la pop-art.

I2 Cfr. Spoto (200I: 226). È stato impossibile identificare il detentore del copyright della fotografia ripresa da http://divinemarilyn.canalblog.com/archives/20Io/o7/30/186931I8.html (consultato il OI.04. 202I). 


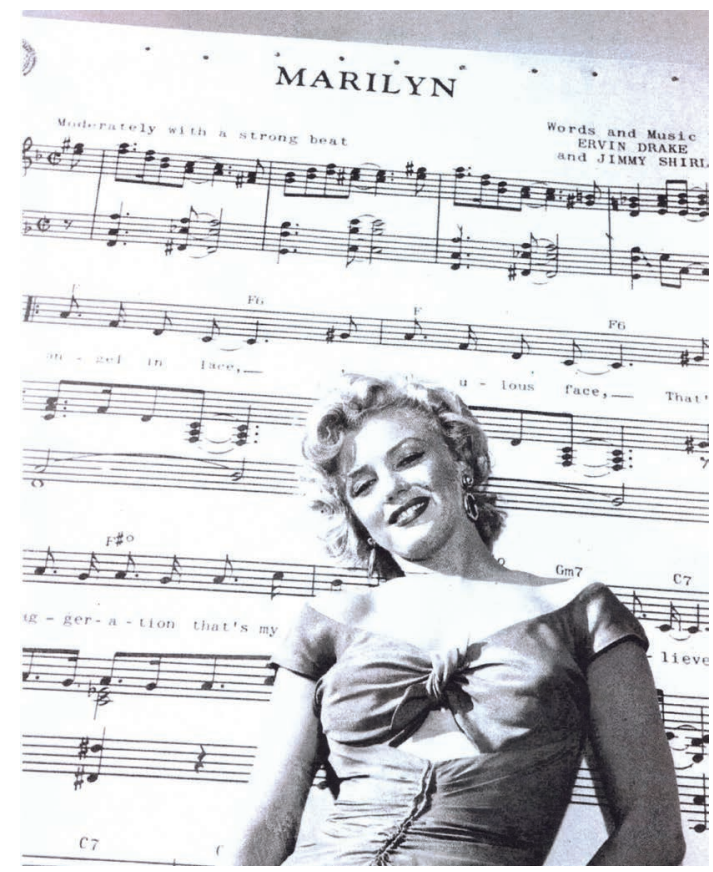

Fig. 3: Marilyn Monroe davanti allo spartito della canzone Marilyn.

Cito l'inizio e la fine del capitolo:

$\mathrm{Ci}$ arrivi soltanto se ci canti. Con ag-ger-a-tions that's my le arrivi proprio lí, cantando, a destra, alla spalla destra appena [...]. È con $G m 7$, invece, che le baci la spalla sinistra, a quella Marilyn. Con $o$ sei piú vicino ancora, lí alla base stessa del collo [...]. Lí c'è anche un bell'accordo di tre note nere tenute bene, con i tre puntini neri. C'è un bell'arco di due note bianche legate, che passano da un la con un re a un si o a un si bem., piano piano. Arrivi a quelle ciocche a destra, cosí [...]. Poi tutto è come è scritto lí su, sotto quella MARILYN maiuscola, tutto spostato, quando è scritto: Moderately with a strong beat (I76-I77).

Verbalizzati dalle istanze di enunciazione "organiche", l'io implicito e il tu implicato, tutti gli elementi sono presentati sullo stesso piano, cioè meccanicamente messi in relazioni di contiguità tra di loro senza tener conto delle loro differenze ontologiche e di profondità: così il corpo della donna può essere toccato dal canto in quanto lettura della notazione musicale scrupolosamente dettagliata, fino al nonsensico «o», parte della sigla $\mathrm{F}^{* 0}$ coperta dal collo, ma che non viene esplicitata. E così la casella non fa che 
differenze ottiche fra «quella Marilyn» corporea e «quella MARILYN maiuscola» del titolo della canzone: fotograficamente, appunto.

\section{7}

Quella Marilyn davanti allo spartito di Marilyn è come l'ultima delle molte sovrapposizioni che caratterizzano Il Giuoco dell'Oca, dall'evocazione di più media e più codici nello stesso contesto alla tensione riflessiva fra elementi discrepanti; dalla sovrapposizione concreta fra la confezione della trottola e la carta di Vautier al paradigma che "blocca" la sequenza. Sono legate tutte alla contestazione del narrare tradizionale attraverso le immagini, fotografiche al livello più profondo. Servono a Sanguineti a radicalizzare la sperimentazione. Infatti, se il gioco dell'avanguardia consiste nell'andare oltre gli altri - i confrères francesi anzitutto -, con il suo Giuoco Sanguineti chiaramente vince la partita.

\section{Bibliografia}

Allasia, Clara, "La testa in tempesta». Edoardo Sanguineti e le distrazioni di un chierico, Novara, Interlinea, 2017.

Bongiorno, Giorgia, «Du jeu de l'oie comme labyrinthe. L'opera aperta d'Edoardo Sanguineti», Revue des Études italiennes, 57, I-2, 20II, pp. I4II49.

Bourdieu, Pierre, Un Art moyen. Essai sur les usages sociaux de la photographie, Paris, Minuit, 1965.

Breton, André, Manifestes du surréalisme, Paris, Pauvert, 1972.

-. Nadja, Paris, Gallimard, 1964.

Eco, Umberto, «L'opera in movimento e la coscienza dell'epoca» (I959), in Balestrini, Nanni - Barilli, Renato et al. (a cura di), Gruppo 63. L'antologia Critica e teoria, Milano, Bompiani, 2013, pp. 749-775.

Genette, Gérard, «Frontières du récit», Communications, 8, I966, pp. I52-I63.

Hempfer, Klaus W., «(Pseudo-)Performatives Erzählen im zeitgenössischen französischen und italienischen Roman», Romanistisches Jahrbuch, 50, I999, pp. I58-I82.

Hertrampf, Marina, «Pour une écriture photographique. Formen und Funktionen photographischer Schreibverfahren bei Alain Robbe-Grillet und Patrick Deville», in Mecke, Jochen (Hg.), Medien der Literatur, Bielefeld, transcript, 20II, pp. 8I-IO2.

Horstkotte, Silke - Pedri, Nancy, «Introduction: Photographic Interventions», Poetics Today, 29, I, 2008, pp. I-29. 
Metz, Christian, «Photography and Fetish», in Squiers, Carol (ed.), The Critical Image: Essays on Contemporary Photography, Seattle, Bay Press, 1990, pp. I55-I64.

Montandon, Alain (éd.), Iconotextes, Paris, Ophrys, 1990.

Nerlich, Michael, «Qu'est-ce qu'un iconotexte? Réflexions sur le rapport texte-image photographique dans La femme se découvre d'Evelyne Sinnassamy», in Montandon I990, pp. 255-303.

Ó Ceallacháin, Éanna, «Making it nouveau. Neoavanguardia Narratives and the French nouveau roman», Italian Studies, 68, 2, 2013, pp. 275-294.

Rajewsky, Irina, Intermedialität, Tübingen-Basel, Francke, 2002.

Rizzarelli, Maria, «Nuovi romanzi di figure. Per una mappa del fototesto italiano contemporaneo», narrativa, 4I, 20I9, pp. 4I-54.

Robbe-Grillet, Dans le labyrinthe, Paris, Minuit, 1959.

Sanguineti, Edoardo, Il Giuoco dell'Oca, Milano, Feltrinelli, 1967.

Sollers, Philippe, Drame, Paris, Seuil, 1965.

Sotgiu, Elisa, «Il Giuoco dell'Oca nella trilogia di Edoardo Sanguineti», Italianistica, XLV, 2, 20I6, pp. I4I-I55.

Spoto, Donald, Marilyn Monroe, New York, Cooper Square Press, 200I.

Wagner, Peter, «Introduction: Ekphrasis, Iconotexts, and Intermediality the State(s) of the Art(s)», in Id. (ed.), Icons - Texts - Iconotexts, Berlin-New York, de Gruyter, 1996, pp. I-40.

Warning, Rainer, Die Phantasie der Realisten, München, Fink, 1999.

—. «Erzählen im Paradigma. Kontingenzbewältigung und Kontingenzexposition», Romanistisches Jahrbuch, 52, 200I, pp. I76-209. 
